\title{
EL EFECTO DE LA COMPETENCIA POLÍTICA SOBRE LA PROVISIÓN DE BIENES PÚblicos locales en MÉXICO*
}

\author{
The Effect of Political Competition on the Provision \\ of Local Public Goods in Mexico
}

\section{IRVIN MIKHAIL SOTO ZAZUETA}

Universidad Autónoma de Sinaloa

\begin{abstract}
RESUMEN
La competencia política tiene efectos similares a la económica en los mercados. Con información de corte transversal referente a elecciones municipales en México, evaluamos el efecto que ella tiene sobre la provisión de bienes públicos locales, tomando en consideración factores socioeconómicos y demográficos. La evidencia sugiere que la competencia política se relaciona positivamente con la tasa de cobertura de servicios públicos municipales, teniendo un impacto mayor en aquellos cuyas condiciones de pobreza y desigualdad son más severas.
\end{abstract}

Palabras clave: competencia política, provisión de bienes públicos, gobiernos locales, elección pública, México, Código Jel: H41, H7, D72

\begin{abstract}
Political competition may have virtuous effects similar to the effect that economic competition has in markets. Using cross-sectional data on local elections in Mexico, we evaluate the effect of political competition on the provision of local public goods, controlling for socio-economic and demographic factors. Evidence suggests that political competition is positively related to the coverage of municipal public services, having a greater effect in those areas with the highest poverty and income inequality conditions.
\end{abstract}

Key words: political competition, provision of public goods, local governments, public choice, Mexico, Jel Code: H41, H7, D72

Este trabajo fue financiado por la Universidad Autónoma de Sinaloa a través del Programa de Fomento y Apoyo a Proyectos de Investigación (2014). Agradecemos los comentarios de dos dictaminadores anónimos que nos ayudaron a mejorar significativamente el documento. Los errores u omisiones que existan son nuestra responsabilidad. 


\section{INTRODUCCIÓN}

Así como la ausencia de competencia en los mercados privados conduce a precios elevados y a una oferta restringida de bienes y servicios, en donde el monopolista obtiene rentas extraordinarias en detrimento del bienestar de los consumidores, algunos investigadores consideran que la falta de competencia política puede tener efectos análogos en la extracción de rentas por parte de los políticos y la eficiencia en la provisión de bienes públicos (Stigler 1972; Becker 1983; Wittman 1989).

Según el punto de vista de estos autores, la competencia política es un mecanismo vertical de rendición de cuentas que genera incentivos para implementar prácticas de buen gobierno y eficiencia en la provisión de bienes públicos, dado que los ciudadanos utilizan su voto como una forma de ejercer control sobre sus representantes.

En esta lógica, Ibarra (2009) afirma que el proceso democratizador experimentado en México desde la década de 1980, trajo consigo la promesa de gobiernos cercanos a la ciudadanía, con respuestas a sus demandas y soluciones a problemas colectivos. Esto implica que la sustitución del sistema de partido hegemónico por uno competitivo habría resultado en una mejor provisión de servicios públicos a cargo de autoridades locales.

Sin embargo, en México, las evaluaciones orientadas a explicar cómo la competencia política ha influido sobre la provisión de bienes públicos muestran resultados mixtos: por un lado, la literatura presenta indicios de que la competencia política ha incrementado el alcance y la fuerza de las políticas públicas locales, así como los efectos positivos en el desempeño gubernamental, por el otro, también existen investigaciones que expresan escepticismo al sostener que la competencia política ha traído nuevos incentivos para que partidos y políticos actúen con oportunismo e incurran en prácticas clientelares. Las elecciones, según arrojan los resultados, no han servido para promover la efectividad de los gobiernos municipales.

Si bien la provisión de bienes públicos se considera un elemento sustancial para mejorar la calidad de vida de los ciudadanos, hay pocos estudios sistemáticos que nos permitan identificar los factores que explican el desempeño de los gobiernos locales en México y estimar con cierta generalidad sus determinantes. En este panorama, la producción de trabajos empíricos adolece de una metodología rigurosa, además de utilizar información correspondiente a la década de 1990.

Por tal motivo, se considera propicio contribuir con un análisis y una metodología que ayuden a evaluar el impacto de los diferentes factores asociados a la provisión de servicios públicos para el contexto mexicano. De ahí que nos hayamos propuesto investigar ¿qué factores influyen en las diferencias municipales de la cobertura de servicios públicos en los municipios mexicanos? 
Más aún ¿bajo qué condiciones económicas, la competencia política contribuye a mejorar el desempeño de los gobiernos locales en México?

La relevancia de este trabajo consiste en la elaboración de un modelo de provisión de bienes públicos en el que se reduce el sesgo por endogeneidad mediante el uso de mínimos cuadrados en dos etapas (2sls). Elaboramos un estudio econométrico con datos de corte transversal para 2.019 de los municipios con información de elecciones locales que tuvieron lugar entre los años 2007 y 2009. Creemos que una de las principales contribuciones de este trabajo a la literatura es la identificación de efectos no lineales de la competencia política sobre la tasa de cobertura los servicios públicos municipales. Encontramos que la interacción de distintos grados de pobreza y desigualdad condiciona el efecto de la competencia política.

El resto de la investigación se estructura de la siguiente manera: en la segunda sección se discute la relación de la competencia política con la provisión de bienes públicos y se revisan los factores que podrían influir sobre esta. En la tercera sección, se definen los conceptos de desempeño de los gobiernos locales y competencia política. En la cuarta, se describe el modelo econométrico, así como la técnica de estimación que se utiliza, mientras en la quinta realizamos el análisis empírico y discusión de los resultados. Finalmente, en la sexta se presentan las conclusiones.

\section{DISCUSIÓN TEÓRICA Y REVISIÓN DE LA LITERATURA}

\section{Competencia política y provisión de bienes públicos}

Las teorías de las finanzas públicas y del federalismo fiscal establecen que la provisión de bienes y servicios públicos juega un papel importante dentro de las funciones del gobierno (Musgrave 1959; Oates 1968). ${ }^{2}$ La descentralización y transferencia de responsabilidades hacia estados y municipios es necesaria para garantizar la adecuada provisión de bienes y servicios públicos. Dado el supuesto que los gobiernos subnacionales y locales son capaces de suministrar eficientemente dichos bienes y servicios, son ellos los que suelen hacerse cargo de esta tarea (Ruíz-Porras y García-Vázquez 2013).

Desde la perspectiva de la economía política, la democracia genera incentivos para implementar prácticas de buen gobierno y eficiencia en la provisión de bienes públicos. Modelos espaciales de competencia política sobre un sitio ideológico unidimensional como los elaborados por Downs (1957) y Black (1958), han demostrado que la competencia entre dos partidos que anuncian sus plataformas electorales de forma simultánea y no cooperativa -donde los ciudadanos únicamente difieren en sus preferencias ideológicas-, produce

De acuerdo a estos autores, otras dos funciones importantes del gobierno serian la estabilización monetaria y fiscal y la redistribución del ingreso y la riqueza entre regiones y ciudadanos. 
políticas públicas óptimas, traduciéndose en una mayor provisión de bienes públicos.

Por otra parte, Stigler (1972) y Wittman (1989) integra al análisis las posibilidades de los políticos para extraer rentas personales. En una analogía con los mercados económicos, Stigler (1972) argumenta que, así como la competencia económica reduce las rentas del monopolista, la competencia política limita los incentivos de ellos para extraer rentas y aumenta la eficiencia en la provisión de bienes públicos. Esto es, dice Wittman (1989), porque el comportamiento oportunista en un contexto competitivo puede ser muy costoso al afectar negativamente sus aspiraciones de reelección.

Los modelos formales desarrollados por Svensson (1998) y Besley et al. (2010) muestran cómo la escasa competencia política genera incentivos para que el Estado ejerza su poder monopólico, extrayendo rentas y suministrando ineficientemente los servicios públicos, sin preocuparse por las intenciones de reelección. Así ocurre con la nula competencia en los mercados económicos, generando ineficiencias en la producción de bienes y rentas extraordinarias para el monopolista.

Para estos estudiosos, la competencia política proporciona mecanismos de rendición de cuentas. Los ciudadanos utilizan el voto para ejercer control sobre sus representantes, seleccionando a aquellos que mejor representan sus intereses y castigando a quienes no tengan un buen desempeño. El no tener segura la victoria, obliga a los candidatos a buscar ofertas políticas que se ajusten a las preferencias de los electores y a implementar prácticas de buen gobierno y eficiencia en la provisión de bienes públicos (Manin et al. 1999).

En una investigación para la India, Chhibber y Nooruddin (2004) encontraron que estados con sistemas de competencia bipartidistas proveen una mayor cobertura de servicios públicos (electricidad y acceso al agua potable) que aquellos con sistemas multipartidista. En el primer sistema, los partidos requieren el apoyo de diversos grupos sociales, por tanto, la mejor estrategia para ganar elecciones es incrementar la cobertura de servicios y bienes públicos. Mientras que en el segundo, la estrategia es la conformación de clubs, ya que solo es necesario movilizar segmentos de población relativamente pequeños para ganar. $^{3}$

Por su parte, Ashworth et al. (2010) evalúan la eficiencia para 308 gobiernos locales de la región norte de Bélgica para el año 2000. Encuentran que la competencia política disminuye la extracción de la renta y la ineficiencia en los gobiernos municipales. La eficiencia indica que el gobierno genera niveles elevados de los bienes públicos con un presupuesto limitado. La legislación brinda a los gobiernos una gran autonomía para impulsar sus propias políticas, 
pueden iniciar cualquier política que promueva los intereses de sus electores y determinar cómo financiarlas, siempre y cuando estas no estén prohibidas explícitamente por la legislación federal.

Para el contexto mexicano, los trabajos de Rodríguez (1998), Hiskey (1999), Beer (2003) y Hecock (2006) evidencian el efecto positivo de la competencia política sobre el desempeño los gobiernos locales durante los noventa. Rodríguez (1998) argumenta que la competencia alentó a los gobiernos locales a poner un mayor énfasis en la eficiencia, adoptar enfoques más tecnocráticos y principios de transparencia en la elaboración de políticas públicas. Asimismo, Hecock (2006) apunta que la competencia política aumenta la sensibilidad de los gobernantes a las demandas sociales. Muestra que una mayor competencia electoral condujo a un aumento del gasto en educación de los gobiernos estatales entre 1999 y 2004.

En contraste, Cleary (2007) y Moreno-Jaimes (2007) afirman que los efectos de la competencia política en México son bastante limitados. Si bien reconocen que ha incrementado el alcance y la fuerza de las políticas públicas locales en México, también advierten que las elecciones competitivas han traído nuevos incentivos para que partidos y políticos actúen con oportunismo.

Cleary (2007), en su análisis para 2.400 municipios de México durante el periodo 1989 a 2000, encuentra que el margen de victoria no tuvo efecto significativo sobre la provisión de bienes públicos (las tasas de cobertura del agua potable y drenaje). Entre los factores que podrían limitar la capacidad del sistema político para producir gobiernos responsables, menciona los problemas del agente-principal, reglas electorales sesgadas y culturas políticas autoritarias. En ambientes competitivos, precisa, los políticos pueden recurrir al clientelismo y al patronazgo para consolidar una base de apoyo sin mejorar las políticas públicas, así como comprar votos o realizar proyectos de alto perfil que no contribuyen al desarrollo o a la mejora de los servicios, tal como el embellecimiento de plazuelas.

Kitschelt y Wilkinson (2007) señalan que la capacidad de la competencia para inducir gobiernos responsables depende del grado de desarrollo económico. Exponen que la competencia política fortalece el clientelismo, especialmente en áreas con altos grados de pobreza y desigualdad, mientras que en países desarrollados estimula una mayor provisión de bienes públicos. Otros problemas de los sistemas democráticos, señala Moreno-Jaimes (2012), podrían ser la inconsistencia de las preferencias ciudadanas en el tiempo y la desigualdad para influir en la toma de decisiones.

Ahora bien, considerando los hallazgos en los distintos entornos institucionales que podrían condicionar los resultados, el interés de la presente investigación es verificar si la competencia política tiene efecto positivo en la provisión de bienes públicos locales en México. Para ello, hemos diseñado una metodología que, desde nuestro punto de vista, resuelve puntos débiles de aquellas utilizadas en trabajos de esta naturaleza. 


\section{Relación de la provisión de bienes públicos con otros factores}

El desempeño de los gobiernos municipales es multifactorial. Tras una revisión de la literatura internacional, incluida por supuesto la competencia política, encontramos más explicaciones acerca de la provisión de bienes públicos locales y el efecto de variables comúnmente aceptadas. Hemos identificado un conjunto de éstas que son importantes predictores, permitiéndonos agruparlas en factores económicos, demográficos y políticos.

Un primer grupo de variables están vinculadas a las características económicas de las comunidades. Desde el trabajo de Lipset (1959), se argumenta que el desarrollo económico está correlacionado con los procesos políticos. El autor sostiene que el bienestar económico de la sociedad -donde relativamente pocos ciudadanos son pobres- es indispensable para desarrollo democrático, porque aumenta la capacidad para la toma de decisiones racionales en el terreno electoral.

En este sentido, Moreno-Jaimes (2007) observa una relación negativa entre la tasa de pobreza y la cobertura de servicios públicos municipales, al expresar que la modernidad socioeconómica ha sido uno de los factores más influyentes en el desempeño de los gobiernos locales. Por lo tanto, se considera que la provisión de servicios públicos en los municipios puede estar en función de la tasa de pobreza, donde deberíamos esperar una relación negativa.

Así mismo, la educación se considera uno de los factores más importante en los procesos democráticos. Soto y Cortez (2012) destacan el papel de esta en la formación de una cultura política que, a su vez, se relaciona con el desempeño del gobierno. Muro (2006), por ejemplo, argumenta que la participación ciudadana es una manifestación de la cultura política que se deriva de la educación cívica de la población. Mientras, en su estudio para las regiones de Italia, Putnam (1993) señala la existencia de una estrecha relación entre la cultura cívica y el desempeño del gobierno.

En teoría, la educación ayuda a los ciudadanos a permanecer informados acerca de los sucesos políticos, crea una población más activa y mejora la capacidad para articular demandas a las autoridades. Específicamente, exponemos que los municipios con niveles altos de educación tendrán un mejor desempeño del gobierno.

La desigualdad en la distribución del ingreso es otra explicación para la provisión de bienes públicos. Las investigaciones han encontrado que existe una relación negativa entre la desigualdad en la distribución del ingreso y la provisión de bienes públicos (Ruiz Euler 2014). Estudios en México demuestran que la desigualdad en la distribución del ingreso aumenta la heterogeneidad de las preferencias de los ciudadanos y dificulta la realización de acuerdos, teniendo un efecto negativo sobre la capacidad de las comunidades para realizar una acción colectiva eficaz (Khwaja 2009; Bardhan y Dayton-Johnson 2007).

Así mismo, reduce la capacidad de las comunidades para articular una demanda efectiva por bienes públicos (Dayton-Johnson 2000). Por consiguiente, 
abordamos el desempeño gubernamental en función de la desigualdad en la distribución del ingreso, donde esperamos una relación negativa.

Otros factores claves que tienen influencia en la capacidad de los gobiernos para proporcionar servicios básicos a los residentes son los de carácter demográfico. Desde hace décadas se ha estudiado la relación del tamaño de las ciudades y la concentración de la población con la eficiencia en la provisión de bienes públicos. Para algunos autores, debido a la prevalencia en los problemas de congestión y la acentuación de los problemas sociales, los costos per cápita en la provisión de servicios públicos pueden ser mayores en las ciudades o municipios más grandes (Gabler 1971).Es decir, reduce la eficiencia en la provisión de bienes públicos, haciendo necesario un mayor gasto relativo para mantener la cobertura de la población. Mientras, investigadores como Buettner y HolmHadulla (2013) han encontrado que las ciudades grandes son más eficientes en la provisión de bienes públicos al presentar economías de escala y reduciendo los costos per cápita. Así, consideramos que la provisión de servicios públicos puede expresarse en función del tamaño de la población.

Por otra parte, Carruthers y Ulfarsson (2003), Nakamura y Tahira (2008) y Hortas y Solé-Ollé (2010) han encontrado que las ciudades compactas y concentradas son más eficientes en la provisión de servicios públicos, pues reducen los costos per cápita del suministro de los mismos. En consecuencia, se plantea la provisión de bienes públicos en el ámbito municipal en función de la densidad poblacional, donde esperamos tenga un efecto positivo en el desempeño. Adicionalmente, medimos la concentración con la proporción de la población que vive en zonas rurales, donde creemos existe una relaciona negativa.

Finalmente, otro factor ligado al desempeño del gobierno en México es la relación partidaria entre los gobiernos municipal y estatal, es decir la yuxtaposición. Hernández y Jarillo (2007) y Pérez-Yarahuán (2007) han demostrado que la distribución de los recursos intergubernamentales de los estados hacia los municipios en México está influenciada por consideraciones políticas.

Muestra de ello son los beneficios para aquellos municipios cuyas autoridades políticas pertenecen al partido del Gobernador del Estado y la transferencia de mayores recursos de los gobiernos estatales hacia municipios con mayor número de votantes en época de elecciones estatales. Por lo tanto, la provisión de bienes públicos depende de la relación partidista entre los niveles de gobierno municipal y estatal. Esperamos que un municipio yuxtapuesto tenga un nivel menor de desempeño de gobierno que los no yuxtapuestos.

\section{Definición de desempeño gubernamental y competencia política}

Cejudo et al. (2009) sostienen que, en los últimos años, ha habido un interés cada vez mayor en la calidad del gobierno como tema de estudio, generándose así una creciente lista de bases de datos que buscan capturar este concepto en 
indicadores comparables geográfica y temporalmente. Sin embargo, apunta Moreno-Jaimes (2012), no existe una definición universalmente aceptada sobre desempeño gubernamental, a pesar de la abundante literatura. En principio, se trata de un concepto multidimensional por las numerosas actividades que los gobiernos realizan.

Cejudo et al. (2009) critican el uso de definiciones amplias que mezclan atributos de instituciones políticas, componentes administrativos y resultados de la gestión gubernamental, debido a que estas definiciones abarcan causas, características y consecuencias del ejercicio de la autoridad. Otro de los problemas, señalan estos autores, estriba en la falta de una diferenciación clara entre los mecanismos de acceso al poder y el ejercicio de la autoridad. Asimismo, cuestionan las definiciones basadas en los efectos esperados, pues señalan que no es válido equiparar los resultados de indicadores de desarrollo económico o social con los atributos de la calidad del gobierno, debido a que los vínculos causales no son directos ni lineales.

Cejudo et al. sostienen que una definición apropiada de calidad del gobierno debiera incluir únicamente atributos relacionados con el ejercicio de la autoridad, así como hacer a un lado lo relacionado con los mecanismos de acceso al poder y sus consecuencias o resultados esperados.

Preocupado por esta problemática, Moreno-Jaimes (2012) coincide en enfocarse en los mecanismos de ejercicio del poder y no en los de acceso. Aun más, propone que la calidad gubernativa se entienda a partir de atributos de imparcialidad en los procesos de ejecución de políticas públicas, la rendición de cuentas y la efectividad ${ }^{4}$.

Para el propósito de esta investigación, adoptamos la definición propuesta por Moreno-Jaimes (2012). Asimismo, siguiendo a Moreno-Jaimes (2007, 2008, 2012) y Cleary (2007), medimos el desempeño del gobierno local en términos de la cobertura de un conjunto de servicios públicos sobre los que tienen una responsabilidad formal o, por lo menos, cierto grado razonable de control. En este sentido, la provisión de bienes públicos es quizás una de las obligaciones más importantes de los gobiernos locales ${ }^{5}$.

Sin embargo, hay limitaciones en los datos que nos impiden adoptar un enfoque integral. Al respecto, Cleary (2007) arguye que la falta de agua, electricidad y alcantarillado son problemas serios en la política municipal. Su importancia, señala Cleary (2007), ha sido muy marcada en diversas encuestas para votantes en entrevistas con funcionarios tanto como los innumerables

Consultar Moreno-Jaimes (2012) para profundizar acerca del concepto de calidad gubernativa y en los conceptos de imparcialidad, responsividad y efectividad de las políticas públicas.

El artículo 115 de la Constitución mexicana estipula que los gobiernos municipales tienen a su cargo la provisión de los siguientes servicios públicos: sistemas de agua potable, drenaje y alcantarillado; alumbrado público; recolección de basura; mercados públicos; cementerios; rastros; calles y parques públicos; policía preventiva y tránsito. 
estudios de caso sobre políticas públicas municipales. Por lo tanto, debemos esperar la priorización de esfuerzos para mejorar la cobertura de estos servicios. Proponemos un indicador expresado de la siguiente forma:

$$
I B P=\frac{\sum_{k=1}^{4} x_{j k}}{4}
$$

Donde $x$ es la tasa de cobertura delos servicios públicos provisto en los municipios. Así, proponemos un indicador de provisión de bienes públicos compuesto por el porcentaje de viviendas habitadas con agua entubada, porcentaje de viviendas habitadas con drenaje, porcentaje de viviendas habitadas con energía eléctrica y porcentaje de viviendas con piso distinto a tierral. El índice toma valores entre cero y uno, siendo la fuente de información el censo general de población y vivienda de 2010 realizado por el Instituto Nacional de Estadística y Geografía (INEGI).

Por otra parte, diversos estudios han tratado de medir teórica y empíricamente la competencia política. Martínez-Silva e Icedo-Aquino (2002) definen a la competencia política como la acción ordenada y simultánea donde dos o más partidos políticos presentan posturas que les permiten contender para ganar cargos de elección popular para sus candidatos, esto bajo ciertos lineamientos y en condiciones de igualdad y libertad.

Particularmente, en el ámbito estatal y local, frecuentemente se utilizan resultados electorales para la construcción de indicadores. Autores como Pacheco (2008) y Besley et al. (2010), en estudios para elecciones estatales de Estados Unidos, definen un indicador de competencia basado en la diferencia en votos de los dos partidos políticos de ese país. Sin embargo, el sistema electoral de Estados Unidos es bipartidista, mientras que la legislación electoral en México permite que haya un sistema multipartidista (García-Vázquez et al. 2012). Por tal motivo, tratar de medir la competencia política, usando el margen de victoria entre dos partidos, plantea serias limitaciones, ya que se deja de capturar los votos obtenidos por el resto de partidos políticos.

Para un contexto multipartidista, se han usado indicadores que miden el tamaño, la concentración o fragmentación de los partidos. Para clasificar sistemas de partidos y explicar algunas de las características de este sistema, Rae (1967) propone la utilización del índice de concentración de Herfindahl $(H)$ aplicado a partidos políticos. Rae desarrolló el Índice de Fragmentación Electoral, $F=1-H$, para medir la competencia política del sistema electoral, donde F toma valores entre cero y 1 . Valores cercanos a la unidad representan competencia política alta, mientras que valores cercanos cercano a cero indican poca o nula competencia.

Posteriormente, Laakso y Taagepera (1979), en su análisis acerca del impacto del número de partidos sobre la estabilidad política en 15 países de Europa Occidental, consideran que una forma más intuitiva de ver la competencia entre partidos es con un índice de partidos efectivos, $N=\frac{1}{1-F}$, donde $\mathrm{N}$ toma el valor de 1 si hay un partido que tenga el 100\% de los votos, pero si todos los partidos 
tienen la misma proporción de votos, $\mathrm{N}$ será igual al número de partidos que participan en la contienda electoral.

Motivados por esta problemática -y con el propósito de reducir el sesgo en la medición de la competencia política en sistemas multipartidistas-, se propone la construcción de un Índice de Competencia Política (ICP) mediante el uso del índice de Herfindahl $(H)$ como el propuesto por Rae (1967). De esta forma, el índice de competencia política en el municipio $j$ se define como:

$$
I C P=1-\sum_{k=1}^{n} \alpha_{i j}^{2}
$$

Donde $\alpha$ es la proporción de votos obtenidos en la elección local para presidente municipal, por cada partido político en los municipios. El ICP tiene un rango de $(0,1)$, donde 0 indica poca o nula competencia (alta concentración del poder político), mientras que 1 indica una alta competencia (o nula concentración del poder). La fuente de información para la construcción de esta variable fue la base de datos sobre elecciones locales publicada por el Centro de Investigación para el Desarrollo AC (CIDAC) en su página electrónica.

\section{METODOLOGÍA}

\section{Especificación del modelo econométrico}

En esta sección se plantea el modelo a estimar, así como la técnica econométrica que se emplea para establecer la relación de la competencia política con el desempeño de los gobiernos locales en México. Retomando las variables identificadas en la segunda sección como el conjunto de características políticas, económicas y sociodemográficas del municipio que podrían influir en la provisión de servicios públicos, proponemos un estudio de corte transversal con información de 2.019 elecciones locales celebradas en México entre 2007 y $2009,{ }^{6}$ donde la variable dependiente $i b p$ es la cobertura de bienes públicos en el municipio $j$, la cual es expresada como una proporción y se encuentra acotada a valores entre cero y uno.

Como lo documenta Moreno-Jaimes (2007, 2008 y 2011), cuando esto sucede, los coeficientes estimados por mínimos cuadrados ordinarios (MCO) no cumple con el criterio de eficiencia, lo que podría dar lugar a predicciones fuera del rango 0-1, obligando a suponer que el efecto de las variables independientes es lineal. Lo razonable sería suponer que el efecto marginal de las variables independientes decae a medida que la tasa de cobertura de servicios públicos se aproxima al 100\%. Papke y Wooldridge (2008) señalan que una las formas de solucionarlo es realizar una transformación tipo log-odd, $\log [\mathrm{p} /(1-\mathrm{p})]$. 
Así, suponemos que los efectos marginales de las variables independientes sobre la provisión de servicios públicos podrían ser no-lineales. Por lo tanto, se considera que el desempeño gubernamental en el municipio $j$ puede expresarse como:

$$
\begin{aligned}
& y_{j}=\log \left[\frac{i b p}{1-i b p}\right]=\beta_{0}+\beta_{1} i c p_{j}+\beta_{2} \text { yuxta }_{j}+\beta_{3} \text { pobreza }_{j}+\beta_{4} \sigma_{j}^{2}+\beta_{5} e_{j} \\
& + \\
& \beta_{6} \text { pob }_{j}+\beta_{7} \text { densidad }_{j}+\beta_{8} \text { rural }_{j}+c_{i}+v_{t}+u_{j}
\end{aligned}
$$

La variable explicativa ICP,es el índice de competencia política en el municipio $j$ que tiene un rango $(0,1)$, donde 0 indica nula competencia mientras que 1 indica una alta competencia política. ${ }^{7}$ La yuxtaposición, yuxta, es una variable dummy que toma valores de 1 si el presidente municipal y el gobernador tienen una filiación política diferente o cero de otra manera. La pobreza representa el porcentaje de población del municipio que se encuentra en situación de pobreza, la educación, $e$, se mide con los años promedio de escolaridad en el municipio.

También añadimos el índice de gini, que mide la desigualdad en la distribución del ingreso $\left(\sigma^{2}\right)$, pob es el tamaño de la población, rural es la proporción de la población que vive en zonas rurales, densidad es el número de habitantes por metro cuadrado. Se incluyen variables dummy para controlar las diferencias regionales del país y el año en que se realizó la elección municipal, denotados como $c$ y $v$, respectivamente, $(i=1,2, \ldots, 5 ; t=2007,2008,2009)$. Además, $u$ constituye el término de error del municipio $j$.

Cabe mencionar que no se incluye el rezago de la variable dependiente como explicativa, debido a que en este tipo de modelos, la introducción de rezagos puede inducir una subestimación de la significancia en los coeficientes del resto de las explicaciones (Achen 2000). Además, la tasa cobertura de servicios públicos en 2005 se correlaciona altamente con la pobreza, la educación promedio y el porcentaje de personas que viven en zonas rurales en 2010, motivo por el cual se pueden generar estimadores segados por multicolinealidad y endogeneidad. Su efecto será capturado por el intercepto, corrigiendo así los problemas potenciales antes señalados.

\section{Técnica de estimación}

Un problema que surge del análisis de la revisión de la literatura es la causalidad y probable endogeneidad, ${ }^{8}$ lo cual se debe al efecto de retroalimentación que

Para convertir los valores estimados log-odds de nuevo en términos porcentuales, invertimos la transformación log-odd mediante la siguiente fórmula:

8 La endogeneidad ocurre comúnmente cuando una ecuación contiene variables dependientes tratadas como independientes que se determinan conjuntamente. Si no se corrige el problema de endogeneidad, se producen estimadores sesgados. 
puede existir entre las variables explicativas y la provisión de bienes públicos. Es decir, el sentido de causalidad entre ambos tipos de variables va en ambas direcciones.

Por ejemplo, de acuerdo a la discusión teórica, la competencia política podría generar incentivos para mejorar el desempeño gubernamental y tener algún efecto sobre provisión de bienes públicos. Asimismo, los ciudadanos tienen la oportunidad de evaluar la gestión gubernamental y decidir si retener o no al gobierno en turno. Es decir, los ciudadanos tienen la posibilidad de utilizar su voto como una forma de ejercer control sobre sus representantes. Por lo tanto, el desempeño del gobierno podría tener efectos sobre la competencia política.

Un supuesto importante de MCO es quela correlación entre la variable explicativa y el término de error debe ser igual a cero. Cuando este supuesto no se cumple, entonces, los parámetros que se obtienen son sesgados e inconsistentes. Por lo tanto, existe un sesgo endógeno en la relación de causalidad entre la provisión de bienes públicos y la competencia política que necesita ser corregido.

Para este propósito, una alternativa es utilizar variables instrumental (IV's). ${ }^{9}$ Existen diversas técnicas para solucionar este problema, por ejemplo mínimos cuadrados en dos etapas (2SLS). Esta técnica estima el valor de los parámetros de la regresión indirectamente: primero se estima la relación entre las variables explicativas y sus instrumentos y luegola información brindada por las IV's se sustituye en la regresión planteada para estimar los parámetros de las variables explicativas. De esta manera, al utilizar variables no correlacionadas con el término de error ni con la variable dependiente, se soluciona el problema antes descrito. ${ }^{10}$

Nuestra variable instrumental se construyó a partir de la observación empírica y de desarrollo teórico. Diversos estudios empíricos y teóricos han establecido la existencia de una relación positiva entre la competencia política y la participación electoral, convirtiendo esta variable en un candidato de IV.

Por otra parte, autores como Putnam (1993) y Verba et al. (1995) sostienen que el desempeño de la gestión gubernamental está en función de la capacidad de los ciudadanos para articular demandas y presionar a las autoridades a través de estrategias amplias de participación política -más allá del voto-como protestas, discursos públicos, cabildeo, acción colectiva o el contacto directo con los funcionarios del gobierno.

En aras de capturar el efecto de la participación política sobre la provisión de bienes públicos, investigaciones empíricas como las realizadas por Cleary

\footnotetext{
Una variable instrumental es aquella variable teórica y empíricamente correlacionada con la variable explicativa que causa el problema de endogeneidad, pero que, al mismo tiempo, no está correlacionada con el término de error del modelo ni con la variable dependiente. Esto último es condición necesaria para eliminar el problema de endogeneidad.

10 Esto es cierto siempre que se cumplan las condiciones descritas más adelante.
} 
(2007) y Moreno-Jaimes (2007) han utilizado la participación electoral variable proxy de la participación política. Sin embargo, la participación política va más allá de asistir a las urnas a expresar el voto. Significa tener un seguimiento de las acciones y promesas de los candidatos, formar organizaciones sociales para articular demandas sociales, etcétera.

De esta manera, la abstención no significa abstenerse de la participación política, sino de votar. De acuerdo con Nohlen (2004), el abstencionismo puede expresar una forma de protesta contra algún aspecto del sistema político o bien puede ser síntoma de apatía. La mayoría de las veces es interpretada como una forma de expresar malestar contra la política en general, ganando así significado político en sentido estricto. La participación electoral es, en el mejor de los casos, un mal proxy de participación política.

Además, Verba et al. (1978) señalan que los individuos más educados y ricos tienden a participar en política con mayor frecuencia que los menos educados y pobres, efectos ya capturados en sus variables de pobreza y educación municipal. Por lo tanto, consideramos que la participación electoral no tiene un vínculo directo con el desempeño gubernamental, volviéndose un buen candidato para el instrumento de la competencia política. Técnicamente, un buen candidato a instrumento debe estar estrechamente relacionado con la variable explicativa (competencia política) y no debe estar correlacionada con la variable dependiente (provisión de bienes públicos).

\section{Datos}

La evaluación de las hipótesis planteadas hace necesario medir con cuidado varios conceptos. En esta sección, se describe la operacionalización de las variables incluidas en el análisis econométrico.

Índice de Provisión de Bienes públicos (ibp). Como discutimos en tercera sección, empleamos un indicador de desempeño gubernamental construido a partir de un conjunto de servicios públicos municipales comúnmente usados en la literatura empírica, sobre los que los gobiernos locales tienen una responsabilidad formal o, por lo menos, un grado razonable de control (ver sección 3).

Índice de Competencia Política (ICP). Basado en la definición del índice de Herfindahl, el índice de Competencia Política en el municipio $j$ se define como ICP $=1-\sum_{k=1}^{n} \alpha_{i j}^{2}$, (Ver sección 3).

Yuxtaposición (yuxta). Se trata de una variable dummy que indica si el presidente municipal estaba yuxtapuesto. Es decir, era de un partido diferente al del gobernador en el periodo de referencia. Toma valores de uno si el presidente municipal y el gobernador tienen una filiación política diferente o cero de otra manera. 
Población en situación de Pobreza (pobreza). Se utiliza el porcentaje de la población en situación de pobreza por municipio estimado por el Consejo Nacional de Evaluación de la Política y Desarrollo Social (CONEVAL). El indicador se basa en las dimensiones económicas y sociales que señala la Ley General de Desarrollo Social (LGDS): ingreso, rezago educativo, acceso a los servicios de salud, acceso a la seguridad social, calidad y espacios de la vivienda, servicios básicos en la vivienda, acceso a la alimentación y grado de cohesión social.

Educación (e). Es medida con el grado promedio de escolaridad de la población de 15 años y más, en el municipio, para el año 2010. Se utiliza como fuente de información el Sistema Estatal y Municipal de Bases de Datos (SIMBAD) del INEGI.

Desigualdad en la distribución del Ingreso $\left(\sigma^{2}\right)$. Para controlar las diferencias por la desigualdad en la distribución del ingreso, usamos el índice de Gini municipal, que se deriva de las diferencias en las áreas de una línea recta que representa la norma teórica de perfecta igualdad y la línea de la distribución del ingreso $\mathbb{\nabla}$ construida con el ingreso corriente per cápita por déciles de hogares. Este índice es un número entre 0 y 1 , en donde 0 se corresponde con la perfecta igualdad y 1 se corresponde con la perfecta desigualdad. Es estimado por el Consejo Nacional de Evaluación de la Política de Desarrollo Social (CONEVAL).

Población (pob). El tamaño del municipio se mide con el logaritmo base 10 de la población del municipio. La fuente de información es el Censo General de Población y Vivienda 2010 realizado por el INEGI.

Densidad poblacional (densidad). Es medida con el número promedio de habitantes por kilómetro cuadrado de cada municipio. La fuente de información es el Censo General de Población y Vivienda 2010 realizado por el INEGI.

Población rural (rural). Se agrega la proporción de la población que vive en zonas rurales. Es decir, el porcentaje de personas que vive en comunidades con menos de 2.500 habitantes. La fuente de información es el Censo General de Población y Vivienda 2010 realizado por el INEGI.

\section{ANÁLISIS EMPÍRICO}

En la presente sección se discuten los resultados del análisis empírico, donde el modelo trata de explicar las tasas de cobertura de servicios en el año 2010 con el nivel de competencia política, otros medios de influencia política y algunas variables de control. En el cuadro 1 podemos observar el desarrollo de cuatro modelos: en la primera columna se muestran los coeficientes de la estimación de la ecuación (1) mediante la técnica 2SLS. Como podemos observar, los resultados presentados son consistentes con algunos trabajos discutidos con antelación y con los estudios internacionales, a la vez que se presentan algunas diferencias. Particularmente, encontramos soporte empírico de una relación positiva entre la competencia política y la provisión de bienes públicos locales en México. 
Cuadro 1: Resultados del modelo econométrico, segunda etapa 2SLS

\begin{tabular}{|c|c|c|c|c|}
\hline & [1] & [2] & [3] & [4] \\
\hline Constante & $\begin{array}{l}2,4044^{* * *} \\
(0,1254)\end{array}$ & $\begin{array}{l}2,9084^{* * *} \\
(0,0908)\end{array}$ & $\begin{array}{l}2,3558^{* * *} \\
(0,1236)\end{array}$ & $\begin{array}{l}0,9337^{* * *} \\
(0,0601)\end{array}$ \\
\hline $\mathrm{ICP}$ & $\begin{array}{l}0,1942^{* * *} \\
(0,0521)\end{array}$ & $\begin{array}{l}0,1808^{\text {*** }} \\
(0,0526)\end{array}$ & $\begin{array}{l}0,2268^{* * *} \\
(0,0485)\end{array}$ & $\begin{array}{l}0,5049^{* * *} \\
(0,0820)\end{array}$ \\
\hline Yuxtaposición & $\begin{array}{c}-0,0359^{* * *} \\
(0,0146)\end{array}$ & $\begin{array}{c}-0,0403^{* * *} \\
(0,0147)\end{array}$ & & \\
\hline Pobreza & $\begin{array}{c}-0,0139^{* * *} \\
(0,0008)\end{array}$ & $\begin{array}{c}-0,0172^{* * *} \\
(0,0005)\end{array}$ & $\begin{array}{c}-0,0137^{* * *} \\
(0,0008)\end{array}$ & \\
\hline Educación $(e)$ & $\begin{array}{l}0,0586^{* * *} \\
(0,0103)\end{array}$ & & $\begin{array}{l}0,0596^{* * *} \\
(0,0103)\end{array}$ & \\
\hline $\operatorname{Gini}\left(\sigma^{2}\right)$ & $\begin{array}{c}-1,4674^{* * *} \\
(0,1903)\end{array}$ & $\begin{array}{c}-1,2545^{* * *} \\
(0,1881)\end{array}$ & $\begin{array}{c}-1,4497^{* * *} \\
(0,1904)\end{array}$ & \\
\hline $\log ($ densidad $)$ & $\begin{array}{l}0,0418^{* * *} \\
(0,0067)\end{array}$ & $\begin{array}{l}0,0470^{* * *} \\
(0,0067)\end{array}$ & $\begin{array}{l}0,0417^{* * *} \\
(0,0067)\end{array}$ & \\
\hline rural & $\begin{array}{c}-0,2397^{* * *} \\
(0,0257)\end{array}$ & $\begin{array}{c}-0,2933^{* * *} \\
(0,0242)\end{array}$ & $\begin{array}{c}-0,2410^{* * *} \\
(0,0258)\end{array}$ & \\
\hline $\log (p o p)$ & $\begin{array}{c}-0,0516^{* * *} \\
(0,0073)\end{array}$ & $\begin{array}{c}-0,0480^{* * *} \\
(0,0073)\end{array}$ & $\begin{array}{c}-0,0532^{* * *} \\
(0,0073)\end{array}$ & \\
\hline 2007 & $\begin{array}{l}0,0813^{* * *} \\
(0,0178)\end{array}$ & $\begin{array}{l}0,0811^{* * *} \\
(0,0180)\end{array}$ & $\begin{array}{l}0,0825^{* * *} \\
(0,0178)\end{array}$ & $\begin{array}{c}-0,1229^{* * *} \\
(0,0268)\end{array}$ \\
\hline 2008 & $\begin{array}{c}0,0107 \\
(0,0296)\end{array}$ & $\begin{array}{c}0,0200 \\
(0,0298)\end{array}$ & $\begin{array}{c}0,0084 \\
(0,0296)\end{array}$ & $\begin{array}{l}-0,1057^{* *} \\
(0,0458)\end{array}$ \\
\hline Noroeste & $\begin{array}{c}0,0689^{* *} \\
0,0333\end{array}$ & $\begin{array}{c}0,0876^{* * *} \\
0,0334\end{array}$ & $\begin{array}{c}0,0718^{* *} \\
0,0333\end{array}$ & $\begin{array}{l}0,03923 \\
(0,0518)\end{array}$ \\
\hline Centro & $\begin{array}{l}0,0315 \\
0,0321\end{array}$ & $\begin{array}{c}0,0583^{*} \\
0,0320\end{array}$ & $\begin{array}{l}0,0315 \\
0,0321\end{array}$ & $\begin{array}{l}-0,0864^{* *} \\
(0,0341)\end{array}$ \\
\hline Occidente & $\begin{array}{l}0,0310 \\
0,0290\end{array}$ & $\begin{array}{l}0,0337 \\
0,0292\end{array}$ & $\begin{array}{c}0,0386 \\
0,02893\end{array}$ & $\begin{array}{c}-0,1368^{* * *} \\
(0,0371)\end{array}$ \\
\hline Sur & $\begin{array}{c}-0,0366 \\
0,0281\end{array}$ & $\begin{array}{c}-0,0362 \\
0,0283\end{array}$ & $\begin{array}{c}-0,0351 \\
0,0281\end{array}$ & $\begin{array}{c}-0,2814^{\text {*** }} \\
(0,0337)\end{array}$ \\
\hline $\mathrm{R}^{2}$ & 0,6652 & 0,6591 & 0,6639 & 0,1474 \\
\hline Obs & 1812 & 1812 & 1812 & 1818 \\
\hline
\end{tabular}

Fuente: elaboración propia con datos de diversas fuentes ${ }^{*} \mathrm{p}<0,1,{ }^{* *} \mathrm{p}<0,05,{ }^{* * *} \mathrm{p}<0,01$. Desviación estándar entre paréntesis

Uno de los resultados más importantes que viene a corroborar la importancia en los procesos democráticos es el efecto positivo de la educación sobre el desempeño gubernamental. El modelo predice que, si todo lo demás permanece igual, un municipio con mayor número de años promedios de educación de la 
población de 15 años y más tendría mayor cobertura de servicios públicos que uno con poca educación.

La educación promedio, al estar inversamente correlacionada con el porcentaje de personas en situación de pobreza, podría estar generando problemas de multicolinealidad. Una forma de solucionarlo es omitir la educación de la ecuación, resultado que se reporta en la columna 2. El signo de los estimadores permanece inalterado y, más aún, el tamaño de los coeficientes cambia de manera poco significativa. Permanece así el nivel de significancia de cada variable y la bondad de ajuste del modelo, motivo por el cual consideramos que ambas estimaciones son consistentes y comparables.

Por otra parte, en la columna 3 se trata de ver el efecto de la competencia política sin su interacción con la yuxtaposición. Si comparamos con los resultados de la columna 1, podemos observar que el efecto de la exclusión de esta variable es capturado - en su mayoría - por el coeficiente de competencia política y el intercepto, manteniéndose tanto la magnitud como la significancia del resto de los estimadores. Finalmente, en la columna 4 tratamos de comprobar si la relación de la competencia política con la provisión de bienes públicos encontrada es consistente o solo es producto de su interacción con el resto de las variables. La $\mathrm{R}^{2}$ ajustada nos indica una bondad de ajuste de esta especificación es del $14,74 \%$.

Después de corregir los posibles sesgos, la principal variable de interés -la competencia política- muestra un efecto en la dirección esperada en todas las regresiones y su coeficiente pasa las pruebas individuales de significancia. Pero la magnitud del impacto depende, en buena medida, de las configuraciones de las condiciones socioeconómicas de los municipios y las diferencias regionales ${ }^{11}$. Con base en las estimaciones realizadas, en el gráfico 1 se muestra la relación entre el índice de gini y el indicador de cobertura de servicios públicos para determinados valores del índice de competencia política usando los valores promedio del resto de las variables explicativas.

Podemos apreciar que, si todo lo demás permanece constante, una mayor desigualdad en la distribución del ingreso se relaciona negativamente con el porcentaje de cobertura de servicios públicos en los municipios, mostrando una pendiente cada vez más pronunciada. Además, el efecto de la competencia política sobre la provisión de bienes públicos en los municipios crece en la medida que hay una mayor desigualdad.

11 Cabe señalar que, debido a las transformaciones log-odd, los coeficientes reportados en el cuadro 1 no pueden interpretarse directamente, aunque los efectos corren en el mismo sentido que se reportan. 
Gráfico 1. Relación entre la distribución del ingreso y la provisión de bienes públicos en México

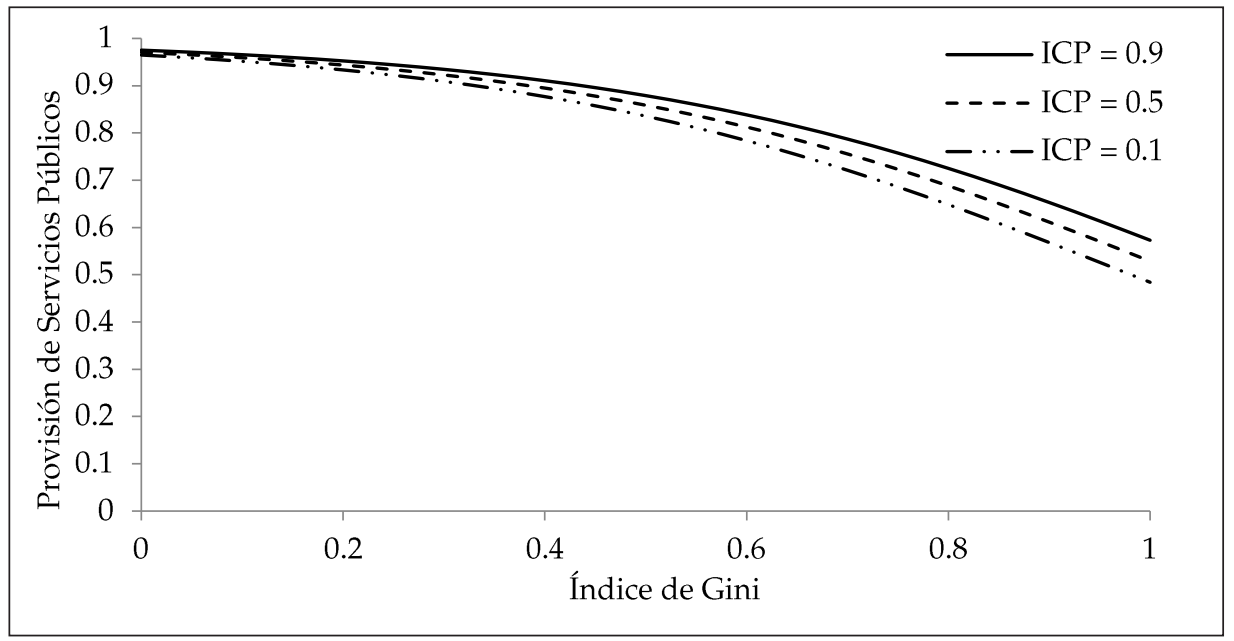

Fuente: elaboración propia realizada con base en las estimaciones mostradas en el cuadro 1

Es decir, el efecto de la competencia política en el desempeño de los gobiernos locales es pequeño en municipios relativamente equitativos, mientras que su impacto es mayor en comunidades donde la distribución del ingreso es relativamente desigual. Existe, aparentemente, un efecto condicionado. Por ejemplo, nuestros hallazgos indican que el efecto marginal de la competencia electoral sobre la provisión de servicios públicos es mayor cuando la desigualdad en la distribución del ingreso y/o la pobreza es alta.

La pobreza es otra de las variables relevantes para el análisis. En el gráfico 2 exponemos la relación encontrada entre la competencia política y el desempeño de los gobiernos locales, tomando en consideración el porcentaje de las personas en situación de pobreza. Podemos ver la existencia de una relación positiva entre ambas variables, pero el efecto es diferenciado a través de los grados de pobreza. Las pendientes nos indican que el efecto de la competencia electoral es limitado cuando los niveles de pobreza son bajos, mientras que este efecto cobra importancia en los niveles más altos de pobreza: en los municipios con alta pobreza, hay una diferencia de casi siete puntos porcentuales entre aquellos que muestran competencia política perfecta respecto de los que presentan concentración política perfecta.

Esto hallazgos muestran que el efecto de la competencia sobre la provisión de servicios públicos no es lineal. Asimismo, podemos ver que la variable dependiente es muy sensible a los niveles de pobreza: la cobertura de servicios públicos para un municipio con bajo grado de carencia sería entre 15\% y 21\% superior que aquellos con altos niveles de pobreza, suponiendo que todo lo 
demás permanece constante y son semejantes en el resto de las condiciones socioeconómicas.

Gráfico 2. Relación entre competencia política y provisión de bienes públicos en México

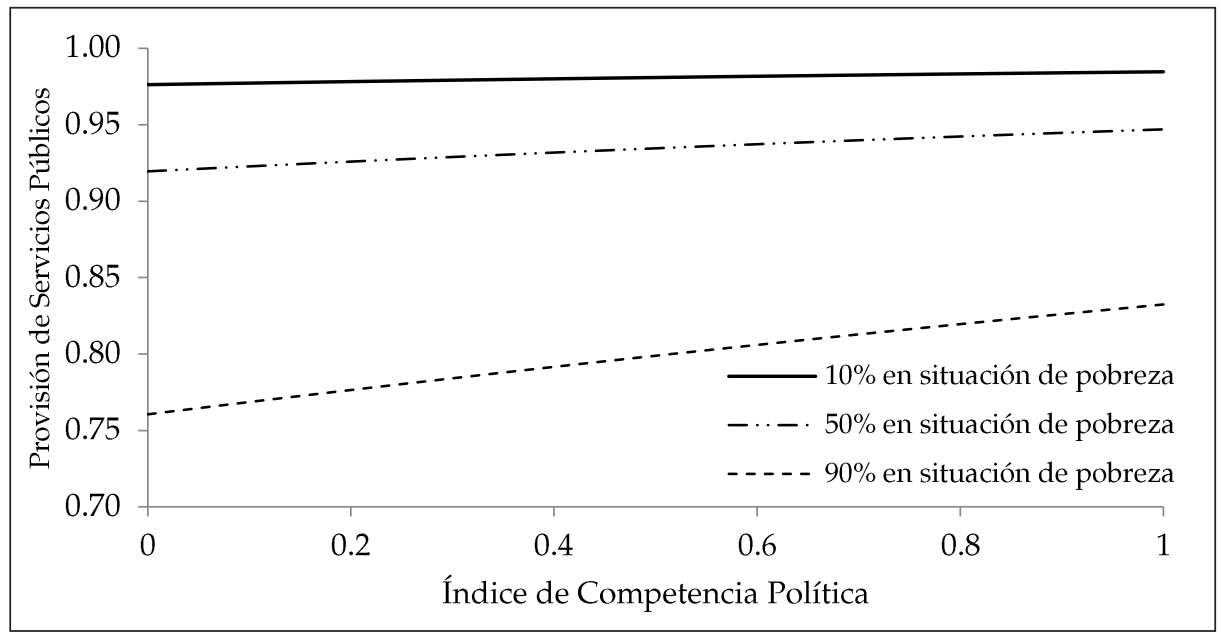

Fuente: elaboración propia realizada con base en las estimaciones mostradas en el cuadro 1

En conjunto, las consecuencias de la educación, la desigualdad en la distribución del ingreso y la pobreza sobre el desempeño gubernamental es consistente con las teorías de modernización política: la modernidad socioeconómica parece ser una condición importante para fomentar la capacidad de respuesta de las autoridades gubernamentales.

Por otro lado, encontramos que la densidad poblacional se relaciona positivamente con nuestro indicador de bienes públicos. Los resultados sugieren que, manteniendo constantes todos los demás factores, los municipios más densamente poblados (menor dispersión) tienen una mayor tasa de cobertura servicios públicos. Es decir, la dispersión geográfica de los ciudadanos tiene un efecto negativo sobre la tasa de cobertura de servicios públicos.

Esto es consistente con los argumentos de Carruthers (2003), Nakamura y Tahira (2008), Hortas-Rico y Solé-Ollé (2010) y con los resultados de MorenoJaimes (2007), quienes señalan que el costo per cápita es menor en comunidades compactas que en aquellas zonas con mayor dispersión geográfica. En efecto, Moreno-Jaimes (2007) apunta que la dispersión geográfica de las personas en el territorio municipal hace que sea más costoso proporcionar agua, saneamiento y drenaje en las zonas aisladas que en zonas de mayor concentración de la población. 
Una aparente contradicción en este trabajo, que resulta no serlo después de revisar la literatura, es la relación negativa del tamaño de la población con la provisión de bienes públicos locales. Para algunos investigadores parecería lógico pensar que el tamaño de las ciudades podría mostrar rendimiento a escala, reduciendo los costos per cápita del suministro de servicios públicos y aumentando las tasas de cobertura. Lo cierto es que este efecto ha sido capturado previamente por la densidad de la población. Otros autores como Buettner y Holm-Hadulla (2013) indican que los costos per cápita en la provisión de servicios públicos podrían ser mayores en las ciudades o municipios más grandes debido a la prevalencia en los problemas de congestión y a la acentuación de los problemas sociales.

Asimismo, se confirma que los municipios con una mayor proporción de personas viviendo en comunidades rurales tienen una cobertura menor, corroborando así la hipótesis de la urbanización. A raíz de los valores arrojados por las dummies de regiones, podemos afirmar que los municipios del sur presentan menores tasas de coberturas de servicios públicos, mientras que la mayor cobertura se presenta en los municipios del noroeste del país.

Finalmente, encontramos que la yuxtaposición es negativa y estadísticamente significativa, sugiriendo que los municipios controlados por un partido distinto al del Gobernador presentan tasas de cobertura ligeramente menores. Sin embargo, esta situación solo representa una diferencia de $1 \%$ en las tasas de cobertura.

En resumen, la evidencia presentada parece soportar la hipótesis de la teoría electoral de la rendición de cuentas, la cual sugiere que el desempeño del gobierno municipal ha sido sensible a un entorno electoral, particularmente a la competencia política. Sin embargo, el efecto está condicionado por las circunstancias socioeconómicas adversas, tales como la pobreza y la desigualdad. Por otra parte, los gobiernos municipales parecen responder más a la influencia de variables socioeconómicas. La cobertura de servicios públicos es significativamente mayor en los municipios que tienen una distribución del ingreso relativamente equitativa, mayor educación y menos pobres.

\section{CONCLUSIONES}

La producción de trabajos empíricos sobre el desempeño gubernamental en México con metodología rigurosa es muy limitada todavía. La presente investigación tiene como objetivo central explicar las diferencias en el desempeño de los gobiernos locales en México. Particularmente, evaluamos el efecto de la competencia política sobre la provisión de bienes públicos locales, donde se consideran factores socioeconómicos y demográficos.

Basados en estudios internacionales, se identificó un conjunto de factores que inciden en la provisión de bienes públicos locales, los cuales se incluyen en el análisis empírico. Asimismo, la técnica de estimación utilizada permite corregir 
problemas potenciales de endogeneidad, heterocedasticidad y sesgo en los estimadores.

Los niveles de competencia política se relacionan positivamente con el desempeño de los gobiernos locales, teniendo un mayor efecto en aquellos con condiciones económicas más severas. Esto es consistente con la teoría electoral de la rendición de cuentas. Las diferencias entre resultados de estudios previos en México -como los elaborados por Cleary (2007) y Moreno-Jaimes (2007, 2008) y los presentados en esta investigación- podrían obedecer a cuestiones metodológicas. $^{12}$

Una primera diferencia es el uso de datos más recientes que los autores antes mencionados. Adicionalmente, tratamos de corregir los sesgos de endogeneidad mediante el uso de variables instrumentales por el método de mínimos cuadrados en dos etapas, aun cuando se utilizan valores rezagados del indicador de competencia política. Otra diferencia importante es la decisión de no incluir el valor rezagado de la variable explicativa, debido a que podría inducir una subestimación de la significancia en los coeficientes del resto de las explicativas, además de causar problemas de endogeneidad y multicolinealidad.

Por otra parte, Cleary (2007) y Moreno-Jaimes (2007, 2008) exponen algunas dificultades para que un régimen democrático se traduzca en un mejor desempeño gubernamental. Una de sus principales razones corresponde al funcionamiento del sistema electoral bajo la presión de instituciones y prácticas que complican los mecanismos de rendición electoral de cuentas, como es el caso de México.

Durante los últimos años, este país ha avanzado indudablemente en la creación de instituciones democráticas que norman la actuación de los funcionarios gubernamentales, el uso de los recursos públicos y los programas sociales. Sin embargo, coincidimos con Moreno-Jaimes (2012) en que habría que liberar al aparato gubernamental de la interferencia excesiva del clientelismo políticoelectoral, del uso indiscriminado de los programas sociales y del empleo burocrático, como mecanismos de control político. Falta avanzar más hacia reformas más profundas, orientadas a promover una efectiva rendición de cuentas de los tres niveles de gobierno.

La provisión de bienes públicos se relaciona con factores como la pobreza, la desigualdad en la distribución del ingreso y la educación. De hecho, el índice de gini y la pobreza resultan ser de las variables más importantes, mereciendo una mayor atención de los académicos sobre la interacción de estas con el desempeño gubernamental.

El tamaño de las ciudades se asocia negativamente con el desempeño de los gobiernos locales. Este resultado es consistente con la argumentación de que las 
jurisdicciones pequeñas son más eficientes en la provisión de bienes públicos. Por otra parte, la densidad de la población muestra una relación positiva, indicando que la provisión de servicios públicos es mayor en las zonas con más concentración poblacional que en aquellas que se encuentran en situación de aislamiento, probablemente debido a diferencias costo-eficiencia. En relación a las zonas urbanas, la cobertura de servicios públicos es mayor que en las rurales.

Es nuestro interés que los resultados de esta investigación contribuyan a profundizar en esta relación y en otras que, por la naturaleza del estudio, no fueron tratadas a profundidad, pues nos hubiesen alejado del propósito de este estudio. Por citar un ejemplo, la causalidad entre el desarrollo socioeconómico y el desempeño del gobierno local implica que pueden tener una relación simultánea: niveles socioeconómicos altos pueden provocar un buen gobierno, pero al mismo tiempo el desarrollo socioeconómico podría ser el resultado, al menos en cierta medida, de un gobierno local eficaz y eficiente.

\section{REFERENCIAS}

Achen, Christopher H. 2000. "Why Lagged Dependent Variables Can Suppress the Explanatory Power of Other Independent Variables". Ponencia presentada en Annual Meeting of the Political Methodology Section of the American Political Science Association, Los Angeles, 20-22 de julio.

Ashworth, John, Benny Gey, Bruno Heyndels y Fanny Wille. 2010. “Political Competition and Local Government Performance: Evidence from Flemish Municipalities". Working Paper. Bruselas, Bélgica.

Bardhan, Pranab y Jeff Dayton-Johnson, 2007. "Inequality and the Governance of Water Resources in Mexico and South India". En Inequality, Cooperation, and Environmental Sustainability, editado por Jean-Marie Baland, Pranb Bardhan and Samuel Bowles. Princeton: Princeton University Press, 98-127.

Beer, Caroline C. 2003. Electoral Competition and Institutional Change in Mexico. Notre Dam: University of Notre Dam Press.

Becker, Gary S. 1983. "A Theory of Competition among Pressure Groups for Political Influence". Quarterly Journal of Economics 98(3): 371-400.

Besley, Timothy, Torsten Persson y Daniel Sturm. 2010. "Political Competition, Policy and Growth: Theory and Evidence from the United States". Review of Economic Studies 77(4): 1329-1352.

Black, Duncan. 1958. The Theory of Committees and Elections. Cambridge: Cambridge University Press.

Buettner, Thiess y Fédéric Holm-Hadulla. 2013. "City Size and the Demand for Local Public Goods". Regional Science and Urban Economics 43(1): 16-21.

Carruthers, John I. y Gudmundur F. Ulfarsson. 2003. "Urban Sprawl and the Cost of Public Services". Environment and Planning B: Planning and Design 30(4): 503-522.

Cejudo, Guillermo, Gilberto Sánchez y Dionisio Zabaleta. 2009. “El (casi inexistente) debate conceptual sobre la calidad del gobierno". Política y Gobierno 16(1): 115-156.

Chibber, Pradeep e Irfan Nooruddin. 2004. “Do Party Systems Count? The Number of Parties and Government Performance in the Indian States". Comparative Political Studies 37(2): 152-187.

Cleary, Matthew R. 2007. “Electoral Competition, Participation, and Government Responsiveness in Mexico". American Journal of Political Science 51(2): 283-299. 
Dayton-Johnson, J. 2000. “Determinants of Collective Action on the Local Commons: A Model with Evidence from Mexico". Journal of Development Economics 62(1), 181-208.

Downs, Anthony. 1957. An Economic Theory of Democracy. New York: Harper and Row.

Gabler, L. R. 1971. "Population Size as Determinant of City Expenditures and Employment Some Further Evidence". Land Economics 47(2): 130-138.

García-Vázquez, Nancy, Miriam G. Gutiérrez y Antonio Ruíz. 2012. "Elecciones municipales y competencia política en Jalisco, 1982-2009". En La Democracia Electora: Contextos, Estructuras y Resultados, México, coordinado por Nancy García-Vázquez. Ciudad de México: El Colegio de Jalisco, 57-89.

Hecock, R. Douglas. 2006. "Electoral Competition, Globalization, and Subnational Education Spending in Mexico, 1999- 2004". American Journal of Political Science 50(4): 950-61.

Hernández Trillo, Fausto y Brenda Jaramillo Rabling. 2007. "Transferencias condicionadas federales en países en desarrollo: el caso del FISM en México". Estudios Económicos, 22(2): 143-184.

Hiskey, Jonathan T. 1999. Does Democracy Matter? Electoral Competition and Local Development in Mexico. Tesis doctoral, Pensilvania: University of Pittsburgh.

Hortas-Rico Miriam y Albert Solé-Ollé. 2010. "Does Urban Sprawl Increase the Costs of Providing Local Public Services? Evidence from Spanish Municipalities". Urban Studies 47(7): 1513-1540.

Ibarra, Juan Fernando. 2009. “Competencia electoral, gasto público y provisión de servicios en municipios mexicanos". En Nuevas tendencias en la teoría y la práctica de la administración y de las políticas públicas coordinado por María del Carmen Pardo. México: El Colegio de México.

Khwaja, Asim. 2009. "Can Good Projects Succeed in Bad Communities?". Journal of Public Economics 93(7-8): 899-916.

Kitschelt, Herbert y Steven Wilkinson. 2007. Patrons, clients, and policies. New York: Cambridge University Press.

Laakso, Markku y Rein Taagepera. 1979. "Effective Number of Parties: A Measure with Application to West Europe". Comparative Political Studies 12: 3-27.

Lipset, Martin Seymour. 1959. "Some Social Requisites of Democracy: Economic Development and Political Legitimacy". American Political Science Review 53(1): 69-105.

Martínez-Silva, Mario y Roberto Icedo-Aquino. 2002. "Alternancia en el Poder". En Diccionario Electoral, 2da. Edición, editado por Mario Martínez Silva y Roberto Icedo Aquino. México: Instituto Nacional de Estudios Políticos A.C.

Manin, Bernard; Adam Przeworski y Susan Stokes. 1999. "Introduction". En Democracy, Accountability, and Representation, edited by A. Przeworski, Manin. Bernard, y Susan. Stokes. New York, Cambridge University Press, 1-25.

Moreno-Jaimes, Carlos. 2007. "Do Competitive Elections Produce Better-Quality Governments? Evidence from Mexican Municipalities, 1990-2000". Latin American Research Review 42(2): 136-153.

Moreno-Jaimes, Carlos. 2008. Democracia electoral y calidad gubernativa: el desempeño de los gobiernos municipales en México. Tlaquepaque: ITESO-UIA Puebla-UIA Torreón.

Moreno-Jaimes, Carlos. 2011. "Is Local Spending Responsive to the Poor? An Appraisal of Resource Allocation and Electoral Rewards in Mexico". Politics \& Policy 39(6): 1021-1052.

Moreno-Jaimes, Carlos. 2012. "El nexo entre calidad gubernativa y elecciones: discusión conceptual y aplicación al gobierno local mexicano". Perfiles Latinoamericanos 39: 59-90.

Muro, Francisco José. 2006. Educación Cívica, Cultura Política y Participación Ciudadana en Zacatecas. México: Plaza y Valdés Editores.

Musgrave, Richard A. 1959. The Theory of Public Finance: A Study in Public Economy. New York: McGraw-Hill.

Nakamura,Kazuyuki y Tahira Masanori. 2008. "Distribution of Population Density and the Cost of Local Public Services: The Case of Japanese Municipalities". Working Paper $\mathrm{N}^{\circ}$ 231. Faculty of Economics, University of Toyama.

Nohlen, Dieter. 2004. "La participación electoral como objeto de estudio". Elecciones 3: 137-157. 
Oates, Wallace E. 1968. "The Theory of Public Finance in a Federal System". The Canadian Journal of Economics 1(1): 37-54.

Pacheco, Juliana. 2008. "Political Socialization in Context: The Effect of Political Competition on Youth Voter Turnout". Political Behavior 30: 415-436.

Papke, Leslie y Jeffrey M. Wooldridge. 2008. "Panel Data Methods for Fractional Response Variables with an Application to Test Pass Rates". Journal of Econometrics 145: 121-133.

Pérez-Yarahuán, Gabriela. 2007. "Social Development Policy, Expenditures, and Electoral Incentives in Mexico". Ibero Forum 2(3): 1-26.

Putnam, Robert D. 1993. Making Democracy Work: Civic Traditions in Modern Italy. Princeton: Princeton University Press.

Rae, Douglas. 1967. The Political Consequences of Electoral Laws. New Haven: Yale University Press.

Rodríguez, Victoria E. 1998. “Opening the Electoral Space in Mexico: The Rise of the Opposition at the State and Local Levels". En Urban Elections in Democratic Latin America, editado por Henry A. Dietz y Gil Shidlo. Wilmington: SR Books, 163-197.

Ruíz-Porras, Antonio y Nancy García-Vázquez. 2013. “La reforma hacendaria y las transferencias en los municipios de Jalisco 2005-2011". Economía Informa 381(julio-agosto): 29-40.

Ruiz Euler, Rogelio Alexander. 2014. "Electoral Competition, Income Inequality and Public Goods: A Subnational Assessment". Tesis doctoral. Berkeley: Universidad de California.

Soto Zazueta, Irvin y Willy W. Cortez. 2012. “El papel de la educación sobre la competencia política en México: un análisis a nivel estatal, 1980-2009". Política y Gobierno 19(2): 243-281.

Stigler, George J. 1972. "Economic Competition and Political Competition". Public Choice 13: 91-106.

Svensson, Jakob. 1998. “Controlling Spending: Electoral Competition, Polarization and Endogenous Platforms". Unpublished manuscript.

Verba, Sidney, Norman H. Nie y Jae-on Kim. 1978. Participation and Political Equality: A Seven-Nation Comparison. Cambridge: Cambridge University Press.

Verba, Sidney, Key Lehman Schozman y Henry E. Brady. 1995. Voice and Equality: Civic Voluntarism in American Politics. Cambridge: Harvard University Press.

Wittman, Donald. 1989. “Why Democracies Produce Efficient Results". Journal of Political Economy 97: 1395-1424. 


\section{APÉNDICE}

Cuadro A1: Primera etapa 2SLS

\begin{tabular}{lccc}
\hline & Coefficients & standard Error & t-statistical \\
\hline Constante & $0.1780043^{* * *}$ & 0.0153254 & 11.61 \\
electoral Participation & $0.6764377^{* * *}$ & 0.0257095 & 26.31 \\
$\mathrm{R}^{2}$ & 0.2767 & & \\
Obs & 1812 & & \\
\hline
\end{tabular}

Fuente: Elaboración propia con datos de diversas fuentes ${ }^{*} \mathrm{p}<0.1,{ }^{* *} \mathrm{p}<0.05,{ }^{* * *} \mathrm{p}<0.01$.

Irvin Mikhail Soto Zazueta es Doctor en Ciencia Económicas-Administrativas por la Universidad de Guadalajara (2009-2012). Actualmente se desempeña como coordinador de investigación en la Facultad de Ciencias Económicas y Sociales de la Universidad Autónoma de Sinaloa. Su línea de investigación se orienta hacia temas de economía política, teoría de la elección pública y el desarrollo económico, habiendo participado en congresos nacionales e internacionales. Correo electrónico: irvin.soto@uas.edu.mx 